\title{
Amphibian macrophage development and antiviral defenses
}

\author{
Leon Grayfer ${ }^{\mathrm{a}}$ and Jacques Robert ${ }^{\mathrm{b} *}$ \\ ${ }^{a}$ Department of Biological Sciences, George Washington University, Washington, DC, USA \\ and \\ ${ }^{b}$ Department of Microbiology and Immunology, University of Rochester Medical Center, \\ Rochester, NY, USA
}

*Corresponding author: $\quad$ Tel.: 585-275-1722; Fax: 585-473-9573; E-mail address:

Jacques_Robert@urmc.rochester.edu (J. Robert) 
Key Words: amphibian · macrophages $\cdot$ myelopoiesis $\cdot$ monopoiesis $\cdot$ ranavirus $\cdot$ colonystimulating factor- $1 \cdot$ interleukin-34

Abbreviations: Arg-1, arginase-1; CSF-1, colony stimulating factor-1; CSF-1R, colony stimulating factor-1 receptor; CFU, colony forming units; FV3, Frog Virus 3; HSCs, hematopoietic stem cells; IL-34, interleukin-34; M-CSF, macrophage-colony-stimulating factor, NF, Nieuwkoop and Faber; PWM-SCM, pokeweed mitogen-stimulated spleen cell medium; rXl, recombinant Xenopus laevis 


\begin{abstract}
Macrophage lineage cells represent the cornerstone of vertebrate physiology and immune defenses. In turn, comparative studies using non-mammalian animal models have revealed that evolutionarily distinct species have adopted diverse molecular and physiological strategies for controlling macrophage development and functions. Notably, amphibian species present a rich array of physiological and environmental adaptations, not to mention the peculiarity of metamorphosis from larval to adult stages of development, involving drastic transformation and differentiation of multiple new tissues. Thus it is not surprising that different amphibian species and their respective tadpole and adult stages have adopted unique hematopoietic strategies. Accordingly and in order to establish a more comprehensive view of these processes, here we review the hematopoietic and monopoietic strategies observed across amphibians, describe the present understanding of the molecular mechanisms driving amphibian, an in particular Xenopus laevis macrophage development and functional polarization, and discuss the roles of macrophage-lineage cells during ranavirus infections.
\end{abstract}




\section{Introduction}

It is becoming evident that akin to other vertebrate species, amphibians rely heavily on macrophage-lineage cells not only for immune defense, but also for homeostasis and tissue remodeling/resorption (Haislip et al., 2011; Nishikawa et al., 1998). Whereas committed macrophage precursors of other vertebrates reside within designated hematopoietic sites (Bartelmez et al., 1989; Garceau et al., 2010; Kriegler et al., 1994), amphibian species appear to vary in their respective hematopoietic strategies and do not always harbor macrophage precursors within designated sites used by other blood cells for development. This is particularly interesting when considering that many amphibians possess two distinct developmental stages: a typically aquatic tadpole stage and a more terrestrial adult one, each with distinct physiological and immunological requirements. Although metamorphosis is generally rudimentary, or even cryptic in urodelian species (e.g. salamanders, newts), in anuran species it is a major developmental transition between two distinct immune systems [reviewed in (Flajnik et al., 1987; Robert and Ohta, 2009)]. In addition, the different ecological niches occupied by tadpole and adult stages are presumably populated by different pathogens, thus representing unique immunological pressures. As such, metamorphosis is likely to have a profound influence on macrophage development and biology. Macrophage-lineage cells are of particular relevance when considering the alarming increase in the morbidity and mortality of amphibian populations worldwide caused by ranavirus infections [large DNA viruses of the family Iridoviridae (Chinchar, 2002; Chinchar et al., 2009; Williams et al., 2005)]. Indeed, there is increasing evidence implicating amphibian macrophages in persistence, evasion and dissemination of ranaviruses, and possibly differences in the interaction of these pathogens with tadpole and adult 
macrophages [discussed below and reviewed in (Chen et al., 2011; Grayfer et al., 2012)]. Thus, it is imperative that we garner greater insights into the ontogeny and functionality of these amphibian innate immune effectors.

The embryonic origins of amphibian hematopoietic precursors have been described in detail elsewhere (Ciau-Uitz et al., 2014; Ciau-Uitz et al., 2010) and will be addressed in passing here. This review addresses the current understanding of amphibian hematopoiesis with a focus on myelopoiesis, and it highlights recent notable findings pertaining to the roles of amphibian macrophages during ranavirus infections.

\section{Diversified sites of hematopoiesis in amphibians}

Vertebrate blood cell precursors differentiate within designated sites of hematopoiesis. Typically, avian and mammalian committed myeloid-lineage progenitors arise from the bone marrow pluripotent populations (Bartelmez et al., 1989; Garceau et al., 2010; Kriegler et al., 1994), whereas teleost fish utilize the head kidney as their designate site of hematopoiesis (Belosevic et al., 2006; Neumann et al., 2000). In amphibians, the sub-cortical (peripheral) liver is generally considered to be the principal hematopoietic site from early development (Chen and Turpen, 1995; Hadji-Azimi et al., 1987; Hadji-Azimi et al., 1990; Nogawa-Kosaka et al., 2011). However, recent findings, combined with older literature suggest that in fact different amphibian species may actually localize their blood cell development to different organs and tissues (Akulenko, 2012; Brunst, 1958; Carver and Meints, 1977; Durand et al., 2000; Golub et al., 2004; Hadji-Azimi et al., 1987; Hadji-Azimi et al., 1990; Lane and Sheets, 2002). In this regard, it is noteworthy that although the amphibian bone marrow is relatively rudimentary and has been largely overlooked as a potential site of hematopoiesis (Hadji-Azimi et al., 1987; Hadji-Azimi et 
al., 1990), it appears that certain amphibian species utilize this site for blood cell development (discussed below).

\subsection{Urodela}

Hematopoiesis in urodela (salamanders and newts) is thought to occur within the liver and spleen of both larvae and adults (Brunst, 1958; Durand et al., 2000; Golub et al., 2004). In contrast, to our knowledge there are currently no published studies implicating the bone marrow of urodeles in blood cell development. Notably, urodelians are invaluable animal models for hematopoiesis research owing to their life-long regenerative capacity, which anurans only possess during a short period before the onset of metamorphosis [reviewed in (Godwin and Rosenthal, 2014)]. Recent work by Lopez et al. (2014) has demonstrated that axolotl spleen cells are very good producers of hematopoietic colony stimulating factors and are a major source of pluripotent hematopoietic stem cells (HSCs). Stimulation of axolotl splenic HSCs with the pokeweed mitogen-stimulated spleen cell medium (PWM-SCM) gives rise to a predominant fraction of erythroid-lineage cells as well as mixed mononuclear and polymorphonuclear myeloid progenitor populations (Lopez et al., 2014). The detection of PWM-SCM-responsive colony forming units strongly suggests that hematopoiesis in axolotls is confined to spleen and liver but absent from bone marrow, thymus, and kidney tissues (Lopez et al., 2014). This is further underlined by the observation that $\mathrm{GFP}^{+}$spleen- and liver-derived hematopoietic cells, adoptively transferred into $\gamma$-irradiated albino axolotl recipients differentiate only within the liver and spleen tissues (Lopez et al., 2014). Furthermore, when two axolotls are connected by probiosis, thus sharing their circulating $\mathrm{HSCs}, \mathrm{GFP}^{+}$cells from one animal home into and populate the liver and spleen of the other animal. Strikingly, irradiated but not extraneously 
reconstituted animals suffer severe anemia, confirming that the liver and spleen indeed serve as sources of hematopoietic precursors and sites of axolotl blood-cell development (Lopez et al., 2014). Although these investigations do not completely rule out the possibility that other axolotl tissues may be contributing to hematopoiesis, they strongly suggest that the reconstituted liver and spleen cell populations are sufficient to circumvent cell depletion resulting from $\gamma$ irradiation.

\subsection{Anura}

The anuran tadpole and adult life stages exhibit distinct physiology and ecological niches that likely include exposure to different potential pathogens. As such it is not surprising that the blood cell development of these life stages is also distinct.

\subsubsection{Tadpole hematopoiesis}

In Xenopus, the embryonic anterior blood island (equivalent to the mammalian yolk sac) that forms soon after neurulation (NF stage 20, 22-23 hours post-fertilization) serves as the initial source of myeloid cells, whereas erythroid and lymphoid blood cell lineages originate from the adjacent posterior ventral blood island (Ciau-Uitz et al., 2000; Kau and Turpen, 1983; Maeno et al., 1985). At this early developmental stage (NF stage 30), primitive myeloid cells called 'myelocytes' comprise the initial blood cells to differentiate within the Xenopus embryo and can be seen migrating throughout the developing organism (Costa et al., 2008). Even before development of functional vasculature, these myelocytes are readily recruited to sites of injury or pathogenic challenge, possibly serving as transient innate immune effector cells. The long-term 
functional roles, modes of action, and ultimate fates of myelocytes during later animal life remain to be defined.

Early on during Xenopus larval development (NF stage 40-46) and subsequent to the establishment of blood circulation, the liver becomes the primary hematopoietic site, responsible for the formation of erythrocytes, leukocytes and B cells (Chen and Turpen, 1995). At this early developmental stage, when the spleen is still absent, expression of a fluorescence marker under the control of cell type-specific promoters in transgenic animals has revealed that these larvae possess complex and diverse populations of myeloid cells including granulocytic and monocytic lineages (Paredes et al., 2015).

Based on Xenopus studies, the sub-cortical (peripheral) liver is generally considered to be the principal anuran hematopoietic site from early development (Chen and Turpen, 1995; Nogawa-Kosaka et al., 2011). However, hematopoiesis in tadpoles of the European common frog, Rana temporaria, appears to be restricted to pronephric interstices of kidneys, and does not occur in liver at all (Meseguer et al., 1985). Similarly, tadpoles of the edible common frog Rana esculenta employ their trunk pronephrous tissue for granulopoiesis (Frank, 1988; Frank, 1989). Interestingly, during embryogenesis and early larval development of the related leopard frog, Rana pipiens, the pronephrous tissue is also involved in hematopoiesis, although myeloid, lymphoid and erythroid lineages are also generated in the liver (Carpenter and Turpen, 1979). More specifically, precursors of these lineages are localized in the endothelia-lined sinusoids found within the sub-capsular liver as well as deeper within the hepatic tissues (Turpen et al., 1979). The respective contribution of kidney and liver to $R$. pipiens hematopoiesis is presently not clear. It is also notable that, as described below, the bone marrow of post-metamorphic Rana spp. may serve as an important site of hematopoiesis (Carver and Meints, 1977). We believe that 
it would be useful to reexamine some of this older work, using more advanced molecular techniques to delineate the exact contributions of respective Rana spp. tissues to the development of distinct blood cell lineages at distinct developmental stages.

\subsubsection{Adult hematopoiesis}

Since urodelians appear to lack bone marrow-mediated hematopoiesis, anurans may represent the first vertebrate phylogenetic group to involve bone marrow in blood cell development. Immunohistological studies of the adult American bullfrog (Rana catesbeianus) have revealed the presence of active hematopoiesis within the vertebrae, femur and finger bone marrow as well as in their kidneys, but not in spleen, liver, gastrointestinal system, lungs tegument and heart tissues (de Abreu Manso et al., 2009). Unlike salamanders, there is no evidence of hematopoietic activity within the spleen and liver tissues of this species. Moreover, bone marrow and kidney HSCs of $L$. catesbeianus serve as precursors to heterophils, basophils, eosinophils, monocytes, erythrocytes and lymphocytes (de Abreu Manso et al., 2009). These observations are interesting since they indicate that the bullfrogs hematopoiesis takes place not only in the kidney, as in some anuran tadpoles (discussed above) and bony fish, but also in the bone marrow, similar to endothermic vertebrates. Other variations on the hematopoietic sites across anuran species are exemplified by the marsh frog Pelophylax ridibundus, whose hematopoiesis occurs in the bone marrow as in the bullfrog, but unlike the bullfrog also in the liver (Akulenko, 2012). As an additional layer of complexity to the already poorly understood process of anuran hematopoiesis, the $P$. ridibundus bone marrow and the liver erythropoiesis and myelopoiesis appear to be subject to seasonal variations (Akulenko, 2012). 
It will be interesting to delineate the respective contribution of these anuran hematopoietic sites to steady state as well as immunologically and physiologically elicited blood cell development.

\section{Monopoiesis in anura}

\subsection{The roles of anuran bone marrow}

With respect to monopoiesis, or the development of macrophage lineage cells, it is noteworthy that in mammals, surface expression of the colony stimulating factor-1 receptor (CSF-1R) on progenitor cell populations is considered a hallmark of commitment to the macrophage lineage (Tagoh et al., 2002). The transcripts and protein levels of the CSF-1R increase to detectable levels from early precursor stages (detected as macrophage colony forming units) through macrophage development and maturation (Bonifer and Hume, 2008; Krysinska et al., 2007; Rosa et al., 2007). Before this commitment, CSF-1 stimulation is insufficient to drive HSCs down the macrophage developmental pathway (Bartelmez et al., 1989; Kriegler et al., 1994), whereas commitment to the mononuclear phagocyte lineage and the coordinated CSF-1R expression renders these progenitors responsive to CSF-1 stimulation and further macrophagelineage specific differentiation (Cecchini et al., 1994; Stanley et al., 1978).

Interestingly, although the sub-cortical liver clearly functions as the primary site of hematopoiesis in adult X. laevis (Hadji-Azimi et al., 1987; Hadji-Azimi et al., 1990; Lane and Sheets, 2002), our recent findings strongly suggest that committed macrophage precursor populations are located within the bone marrow (Grayfer and Robert, 2013). This is evidenced by the ability of a recombinant $X$. laevis CSF-1 (rXlCSF-1) to stimulate bone marrow cells to proliferate, to form colonies, and to differentiate into cells bearing hallmark macrophage 
morphology and intense CFS-1R gene expression. By contrast, at least under our experimental conditions, $\mathrm{r} X$ lCSF-1 treatment of $X$. laevis sub-capsular liver-derived cells does not result in any observable changes. Furthermore, whereas a distinct bone marrow cell population exhibits rXlCSF-1 binding, cells derived from the adult $X$. laevis peripheral liver fail to bind to this monopoietic growth factor.

Interestingly, upon infection with the ranavirus Frog Virus 3 (FV3, discussed in detail in section 4), X. laevis adults exhibit increased CSF-1R gene expression in kidneys, which is consistent with the recruitment of mononuclear phagocytes (bearing the CSF-1R) to this site of prominent FV3 replication (Grayfer et al., 2015). Moreover, while FV3-infected X. laevis tadpoles increase their splenic and hepatic CSF-1R gene expression, the FV3-challenged adult frogs do not (Grayfer et al., 2015), suggesting that these tadpole and adult organs have distinct roles in monopoiesis. Indeed, although FV3 does not substantially disseminate into the bone marrow (Grayfer and Robert, 2013), CSF-1R gene expression significantly increases within this site during infection (Grayfer et al., 2015). This is consistent with an active involvement bone marrow in $X$. laevis monopoiesis. The robust upregulation of CSF-1R mRNA levels in the bone marrow, induced by stimulation with heat-killed E. coli further substantiates this possibility (Grayfer et al., 2015). Based on the literature and our recent studies, we predict that several adult $X$. laevis organs including the spleen, liver and bone marrow are involved in monopoiesis. We propose that the final steps of these processes are restricted to the bone marrow, while the subcapsular liver and/or spleen tissues supply the pre-committed HSCs that give rise to definitive myeloid populations.

It is intriguing that the development of other adult X. laevis immune cells, such as B lymphocytes, occurs in the spleen and liver tissues but not within the bone marrow (Hadji-Azimi 
et al., 1990; Marr et al. 2007). This implies that, for presently unknown physiological reasons, the final steps of $X$. laevis monopoiesis are segregated into the bone marrow and away from the development of other blood cell subsets. It is particularly notable that more terrestrial anuran amphibian species, such as those belonging to the genus Rana, utilize the bone marrow for erythropoiesis (Carver and Meints, 1977), whereas the Xenopus liver periphery is utilized towards this end (Nogawa-Kosaka et al., 2011). Presumably, involvement of the bone marrow in blood cell development represents a co-evolutionary adaptation, paralleled with the progressive adaptation towards terrestrial life and marked by a move from hepatic to bone marrow-mediated hematopoiesis. In this regard, it is notable that the X. laevis bone marrow is devoid of a central venous system and must rely on sub-endothelial veins to drain this site (Tanaka, 1976). By contrast, the femoral venous systems of terrestrial anurans, such as that of the American bullfrog (Rana catesbiana), possess significantly more developed sub-endothelial venous plexus, which are connected to primary arteries within the bone marrow. This vascularization is reminiscent of that seen in the mammalian bone marrow and coincides with more pronounced bone marrowmediated hematopoietic activity (Tanaka, 1976). This suggests that from a phylogenetic perspective, the increased roles of bone marrow in vertebrate hematopoiesis are perhaps more dependent on the development of effective marrow vascularization rather than the formation of marrow fat. This is intuitive, considering that more developed vasculature would further facilitate hormonal intercommunication of any hematopoietic bone marrow with the rest of the organism and would be more amicable to HSC egress from this site.

The notion that the evolution of sinusoidal architecture lends to more advanced hematopoiesis can be extended to hepatic hematopoiesis as well. Fascinatingly, a comparative histological study involving 46 amphibian species revealed that urodelians and anurans exhibit 
progressively more developed hepatic vascularization combined with more intricate hematopietic sites within the connective tissues and in the perihepatic subcapsular liver (Akiyoshi and Inoue, 2012). Notably, while the more aquatic anuran species of the Bombina and Xenopus genera exhibit hepatic hemaopoietic tissue surrounding this vascular architecture, other, more terrestrial anuran species do not appear to possess hepatic hematopoietic tissues (Akiyoshi and Inoue, 2012). Whether the above reflects amphibian physiological adaptations and/or phylogenetic origins (i.e., the Xenopus, Bombina and Rana belong to very distinct phylogenetic clades), the association between developed tissue vascularization and hematopoiesis in the liver or the bone marrow merits further investigation.

\subsection{Evolution of colony stimulating factor-1}

Monopoiesis is controlled through binding of the principal macrophage growth factor, colony-stimulating factor-1 (CSF-1, macrophage-colony-stimulating factor, M-CSF; Garceau et al., 2010; Hanington et al., 2007; Pixley and Stanley, 2004; Wang et al., 2008) to its cognate receptor (CSF-1R; Dai et al., 2002), which is almost exclusively expressed on committed macrophage precursors and derivative phagocyte populations (Guilbert and Stanley, 1980; Lichanska et al., 1999). It is noteworthy that birds and mammals possess a single, alternatively spliced mRNA transcript, giving rise to membrane-bound and secretory forms of CSF-1 (Manos, 1988; Rettenmier and Roussel, 1988). By contrast, teleost fish possess two distinct CSF-1 genes that do not appear to be alternatively spliced (Wang et al., 2008). It is presently unknown whether the distinct fish molecules encoded by these two CSF-1 genes have assumed the respective biological roles conferred by the mammalian CSF-1 splice variants. 
In mammals, CSF-1R gene expression increases progressively during mononuclear phagocyte development (Stanley et al., 1997), while the ligation of CSF-1R by CSF1 is critical to macrophage biology and immunity (Bober et al., 1995; Karbassi et al., 1987; Munn and Cheung, 1995; Sweet and Hume, 2003). Thus, CSF-1R gene expression serves as a reliable marker in the study of macrophage development and biology.

Although, the respective CSF-1R genes of diverged vertebrate species share poor sequence identities (especially in the extracellular domains), all the gene products, including those of X. laevis possess hallmark CSF-1R features including 5 putative immunoglobulin domains, structurally conserved cysteine residues and a disrupted tyrosine kinase domain (Grayfer et al., 2015). Notably, the intracellular catalytic tyrosine kinase domains of these respective CSF-1R proteins are remarkably conserved, presumably owing to the evolutionary pressure to retain signaling molecule binding sites, which CSF-1R shares with many other intracellular pathways. In contrast, the extracellular portions of CSF-1R molecules have significantly diverged with evolutionary time. This likely reflects selective pressure to facilitate the binding of respective cognate CSF-1 ligands, which also display low amino acid sequence conservation. Notably, the fish, amphibian, avian, reptilian and mammalian CSF-1Rs all phylogenetically branch in respectively separate clades. As many aspects of macrophage biology appear to be distinct across divergent species, it may well prove that the distinct CSF-1 ligand and receptor systems contribute to these differences.

Inspection of the fully sequenced genome of $X$. laevis and X. tropicalis has revealed that unlike fish, there is only one CSF-1 gene per Xenopus genome (Grayfer and Robert, 2013; Grayfer et al., 2015). This difference is further supported by analysis of vertebrate CSF-1 gene synteny. In zebrafish, the two CSF-1 genes are located on distinct chromosomes, each flanked by 
syntenic genes, which are also found within the locus of the single mammalian CSF-1 gene and within a single $X$. tropicalis gene scaffold (Grayfer and Robert, 2013). However, in contrast with mammals, no CSF-1 transcript splice variants could be identified in $X$. laevis. All attempts, including conventional and RACE-PCR on cDNA templates derived from healthy as well as immunologically challenged tadpole and adult X. laevis detected only single transcripts (Grayfer and Robert, 2013). It is notable that the N-terminal 150 residues of the mammalian CSF-1 proteins, which are retained irrespective of splice variation, are sufficient to confer the biological activity mediated by this molecule (Koths, 1997; Taylor et al., 1994). Since it appears that Xenopus have adopted an unusual monopoietic strategy compared to other vertebrate species (i.e. macrophage developing in the bone marrow, away from general hepatic hematopoiesis), it is possible that the presence of a single CSF-1 gene, not subject to alternative splicing is also a Xenopus peculiarity. As a representative amphibian species, X. laevis holds an evolutionarily intermediate position between teleosts, which possess two un-spliced CSF-1s and mammals, encoding a single spliced CSF-1 molecule. Thus, it is conceivable that amphibians have adopted some hybrid of these two respective CSF-1 producing strategies. This notion can be resolved by the characterization of CSF-1 genes in other amphibians, especially terrestrial frogs such as Rana spp..

\subsection{Interleukin-34 as possible sources of macrophage functional heterogeneity}

The functional heterogeneity of macrophage-lineage cells is presently defined by two polarization states:. The classically activated/inflammatory (M1) macrophages produce multiple proinflammatory mediators and participate in an array of antimicrobial responses; whereas alternatively activated/regenerative (M2) macrophages produce immunosuppressive and 
angiogenic compounds and facilitate the resolution of inflammatory sites [reviewed in (Zhou et al., 2013)]. It should be emphasized that these M1/M2 functional subsets represent opposite boundaries of a continuum of possible functional states. Notably, the mammalian CSF-1 contributes to the this functional heterogeneity of macrophage-lineage cells by polarizing macrophages towards the M2 phenotype (Hamilton et al., 2014), whereas the teleost fish CSF-1 appears to skew macrophages towards an M1-like state [reviewed in (Hodgkinson et al., 2015)]. Intriguingly, the interleukin-34 (IL-34) cytokine, which bears no sequence identity with CSF-1, also binds to, and activates with CSF-1R in mammals, thereby contributing to monopoiesis (Chihara et al., 2010; Liu et al., 2014; Ma et al., 2012; Droin and Solary, 2010; Lin et al., 2008; Wei et al., 2010). IL-34 appears to be crucial to the development and regulation of mammalian osteoclasts (Baud'huin et al., 2010; Chen et al., 2011), microglia (Greter et al., 2012), Langerhans cells (Greter et al., 2012; Wang and Colonna, 2014), and B cell-stimulating myeloid cells (Yamane et al., 2014). We speculate that since CSF-1 serves as one facet of macrophage functional polarization (Hamilton et al., 2014), IL-34 may additionally increase the functional spectrum of mononuclear phagocytes.

\subsection{X. laevis tadpole colony-stimulating factor-1- and interleukin-34-derived macrophages}

The identification of CSF-1 and IL-34 genes in Xenopus has provided us with an opportunity to investigate the involvement of these cytokines in promoting macrophage functional heterogeneity in a non-mammalian species. The respective role of CSF-1 and IL-34 was examined in $X$. laevis by producing recombinant forms of these proteins ( $\mathrm{r} X l \mathrm{CSF}-1, \mathrm{r} X l \mathrm{IL}-$ 34) and assessing their roles in tadpole monopoiesis (Grayfer and Robert, 2014). We found that peritoneal macrophages derived by intraperitoneal injection of $\mathrm{rXlCSF}-1$ possess significantly 
greater phagocytic capacities than those derived with $\mathrm{r} X l \mathrm{IL}-34$. The peritoneal macrophages elicited with $\mathrm{rXlCSF}-1$ are also more susceptible to infection with FV3 than cells elicited with rXlIL-34 (Grayfer and Robert, 2014). Indeed, despite being similarly permissive to viral entry during in vitro infection, rXlIL-34-derived macrophages are more potent in preventing viral replication than $\mathrm{r} X$ lCSF-1-derived phagocytes (Grayfer and Robert, 2014). Moreover, when $X$. laevis tadpoles are administered with $\mathrm{rXlCSF}-1$ prior to $\mathrm{FV} 3$ challenge, they more rapidly succumb to FV3 infections (Grayfer and Robert, 2014). In contrast, pre-stimulation of tadpoles with rXlIL-34 prior to FV3 infection significantly extends animal survival times (Grayfer et al., 2014). Presumably, the administration of $\mathrm{rXlCSF}-1$ expands and polarizes a subset of tadpole macrophages, which in turn enhances tadpole susceptibility to this pathogen. Conversely, rXlIL34 enriches tadpoles for macrophage population(s) that are resistant to FV3, as observed in vitro. Consistent with this notion, tadpole macrophages derived with rXlIL-34, but not those derived with $\mathrm{rXlCSF}-1$, express higher levels of a type I interferon gene, which is critical for $X$. laevis anti-ranaviral defenses (Grayfer et al., 2014).

Considering the importance of Xenopus IL-34 in the polarization of potent anti-FV3 macrophages, it is intriguing that FV3 infection does not trigger IL-34 gene expression in tadpole kidneys (the main site of FV3 replication), whereas this pathogen upregulates CSF-1 gene expression at this site.. This suggests that tadpole susceptibility to FV3 may in part stem from the absence of, or inadequate recruitment of this important innate immune cell population to the active sites of infection within their kidneys.

\subsection{X. laevis adult colony-stimulating factor-1- and interleukin-34-derived macrophages}


As described above, CSF-1 contributes to macrophage heterogeneity, while the roles of IL-34 remain to be fully defined. To extend upon our IL-34/CSF-1 studies in tadpoles and in an effort to delineate possible roles of IL-34 in ectothermic vertebrates, we characterized adult $X$. laevis bone marrow- and peritoneum-derived $\mathrm{rXlCSF}-1$ and $\mathrm{rXlIL}-34$ macrophages. As in tadpoles, these cytokines promote morphologically and functionally distinct adult X. laevis macrophage populations (Grayfer and Robert, 2015). Adult bone marrow and peritoneal macrophages derived by $\mathrm{rXlCSF}-1$ stimulation are more phagocytic and express greater levels of the iNOS gene, which is reflected in substantially more robust nitric oxide production by these cells. In contrast, adult $X$. laevis rXlIL-34-derived peritoneal macrophages exhibit greater transcript levels of arginase- 1 as well as the NADPH oxidase catalytic subunits, p67 ${ }^{\text {phox }}$ and gp91 $1^{\text {phox }}$, which is consistent with the greater respiratory burst capacities of these cells. Moreover, akin to tadpole macrophages, adult X. laevis $\mathrm{rXlCSF}-1$ macrophages are highly susceptible to FV3, whereas the $\mathrm{rXlIL}-34$ macrophages possess potent anti-FV3 activity. Interestingly, the adult $X$. laevis $\mathrm{r} X l \mathrm{IL}-34$ macrophages do not express substantially greater type I interferon levels, whereas their anti-FV3 activity appears to be dependent of their robust reactive oxygen responses.

Considering our findings regarding the dichotomous roles of frog CSF-1 and IL-34 cytokines, it is interesting that the binding sites of the mammalian IL-34 and CSF-1 on CSF-1R are distinct and result in unique ligand-receptor conformations and downstream signaling events (Chihara et al., 2010; Liu et al., 2014; Ma et al., 2012). It is equally noteworthy that these cytokines exhibit differential tissue expression across a range of vertebrate species (Chemel et al., 2012; Eda et al., 2011; Grayfer and Robert, 2014; Greter et al., 2012; Hwang et al., 2012; 
Wang et al., 2013; Wei et al., 2010). All these findings are consistent with CSF-1 and IL-34 promoting disparate macrophage functions in X. laevis studies.

In mammals, M1 inflammatory macrophages are characterized by their elevated capacities to undergo respiratory burst and nitric oxide antimicrobial responses; whereas the M2 polarized phagocytes are characterized by their elevated arginase activity (Gordon and Martinez, 2010; Gordon and Taylor, 2005; Mantovani et al., 2002; Mills, 2012). Since macrophage polarization is highly dynamic and reversible (Guiducci et al., 2005; Saccani et al., 2006), these somewhat rigidly defined criteria undoubtedly represent extremes of an as-of-yet poorly defined spectrum of macrophage functional plasticity. It is thus compelling that the same cell-signaling pathways often culminate in M1 and M2 macrophage types [reviewed by (Wang et al., 2014)]. Although not easily categorized according to the M1/M2 classification, X. laevis CSF-1- and IL34-derived macrophages have distinct transcriptional profiles and antimicrobial capacities. For example, enhanced arginase-1 or arginase- 2 activity antagonizes the pro-inflammatory nitric oxide response and serves as a marker of M2 alternatively polarized macrophages in mammals and fish, respectively (Joerink et al., 2006a; Joerink et al., 2006b). Intriguingly, the X. laevis IL34-derived macrophages possess elevated arginase-1 gene expression and yet also exhibit potent respiratory burst responses, typical of mammalian M1 macrophages. It is notable that IL-34 is found across all vertebrates (Garceau et al., 2010; Gow et al., 2012; Gow et al., 2013; Wang et al., 2013). In fact, this cytokine may even predate CSF-1, considering the presence of IL-34 and absence of CSF-1 in the elasmobranch genome (Venkatesh et al., 2014). We believe that further investigation into the respective roles of CSF-1 and IL-34 in non-mammalian vertebrate macrophage monopoiesis will enrich and redefine our present understanding of the evolutionary basis and functional heterogeneity seen across vertebrate macrophage populations. 


\section{Roles of amphibian macrophages during ranavirus infections}

Ranaviruses (family Iridoviridae) are large double stranded DNA viruses that have become major amphibian pathogens (Chinchar, 2002; Chinchar et al., 2009; Williams et al., 2005). Extensive population die-offs caused by these infectious agents are thought to significantly contribute to the decline of amphibian species worldwide [reviewed in Chinchar, 2002; Grayfer et al., 2015]. Increasing evidence suggests that amphibian macrophage-lineage cells are critical to anti-ranaviral immunity as well as to the infection and dissemination strategies. In particular, recent findings indicate that the ranavirus Frog Virus 3 (FV3) infects, persists and disseminates by infiltrating frog mononuclear phagocytes (Morales et al., 2010; Robert et al., 2007) and that distinct macrophage-lineages are involved in viral clearance (Andino et al., 2012; Grayfer and Robert, 2014; Morales et al., 2010).

\subsection{Amphibian macrophage vectors of ranaviral persistance and dissemination}

FV3 infection of $X$. laevis provides an excellent experimental platform for studying the ranavirus-amphibian host immune interactions. Notably, converging lines of evidence from our past and current work suggest multiple important roles for X. laevis macrophages during FV3 infections. For example, FV3 becomes quiescent within $X$. laevis adult macrophages and is detectable up to several months subsequent to the clearance of the primary infection (Robert et al., 2007). This is of relevance considering that terminally differentiated long-lived macrophages represent an excellent outlet for pathogen persistance and dissemination.

Indeed, this notion has been by-and-large empirically supported, whereby transmission electron microscope analysis of FV3-infected X. laevis peritoneal leukocytes, unequivocally 
revealed icosahedral viral particles in peritoneal leukocytes bearing macrophage morphology (Morales et al., 2010). These FV3-infected phagocytes exhibit accumulated pools of assembled viral particles, suggesting that frog macrophages serve as FV3 reservoirs, likely towards dissemination and reminiscent of the HIV-macrophage relationship, wherein large pools of formed viral particles accumulate intracellularly, but are not released until the infected macrophages come into contact with T cells, which are the primary cellular HIV targets (Coiras et al., 2009; Goodenow et al., 2003; Gousset et al., 2008; Groot et al., 2008).

Intraperitoneal inoculation of adult X. laevis with FV3 results in leukocyte recruitment, with macrophage-like cells comprising a large proportion of these cells (Morales et al., 2010). These phagocytes express both hallmark macrophage inflammatory genes (TNF $\alpha$, IL-1 $\beta$ and Arginase-1; Morales et al., 2010) as well as the reliable macrophage-lineage marker, CSF-1R (Grayfer and Robert, unpublished observations). Interestingly, while the FV3 genome is detectable within these peritoneal phagocytes for up to 21 days post infection, the virus appears to become predominantly transcriptionally silent as FV3 early and late genes are no longer detectable at later infection times (Morales et al., 2010). Presumably, the viral genome is quiescently maintained within frog phagocytes and may be transcriptionally reactivated, pending appropriate physiological cues. Furthermore, we have recently found that in vitro FV3-infected $X$. laevis macrophages may harbor transcriptionally quiescent, infectious virus for as long as several months (Grayfer and Robert, unpublished observations).

\subsection{Reactivation of macrophage-residing quiescent Frog Virus 3}

With infrequent exceptions, $X$. laevis peritoneal phagocytes typically do not possess detectable FV3 DNA or transcripts one month after viral infection (Robert et al., 2014). By 
contrast, FV3 genomic DNA and active viral gene expression are detected in as much as sixtyseven percent of previously infected frogs subsequent to their inflammatory stimulation by ip administration of heat-killed E. coli (Robert et al., 2014). Moreover, immunofluorescence microscopy using Abs against an FV3 gene product required for FV3 replication (53R) and a known X. laevis macrophage marker [HAM56; (Nishikawa et al., 1998)] indicates that indeed these reactivated peritoneal populations consist of macrophage-lineage cells bearing productive FV3 replication (Robert et al., 2014).

As evidenced in the above findings and described in greater detail above, anurans possess macrophage populations that are highly susceptible to FV3 (CSF-1-derived) as well as mononuclear phagocyte subsets that are important towards the clearance of this pathogen (IL-34derived).

\section{Concluding remarks}

It is evident from the literature described above that much remains to be elucidated regarding the monopoietic strategies of amphibian species and the roles of distinct macrophage populations during amphibian immune responses. In the face of the global amphibian decline, it is imperative to more fully examine the disparate hematopoietic/monopoietic strategies in phylogenetically and physiologically divergent amphibian species. We believe that this will bring us closer to understanding why there is such variability in susceptibility/resistance to pathogens like ranaviruses across amphibian species. Likewise, a better characterization of differences in the antiviral efficacies of macrophage populations between susceptible and resistant amphibian hosts, including the proportions of CSF-1 and IL-34-derived phagocytes, 
should decipher the respective contributions of these effector cell populations to anti-ranaviral defenses.

To date research into amphibian immunity mainly represents work performed in few key animal models (e.g., Xenopus, axolotl). We hope that through this review we have successfully conveyed the breadth of distinct hematopoietic and immunological strategies seen in different amphibian species. It is our conviction that study of macrophage function should be extended to non-model amphibian species in order to close the gap in our understanding of the complex roles of macrophage in immune defenses against ranaviruses.

\section{Acknowledgements}

L. Grayfer thanks the Howard Hughes Medical Institute-Life Sciences Research Foundation for postdoctoral research support. J. Robert research support: 2 R24 Al 059830-10 from the NIH, and IOS-0923772 from the NSF. 


\section{References}

Aggad, D., Stein, C., Sieger, D., Mazel, M., Boudinot, P., Herbomel, P., Levraud, J. P., Lutfalla, G., Leptin, M., 2012. In vivo analysis of Ifn-gamma1 and Ifn-gamma2 signaling in zebrafish. J Immunol. 185, 6774-82.

Akiyoshi, H., Inoue, A. M., 2012. Comparative histological study of hepatic architecture in the three orders amphibian livers. Comp Hepatol. 11, 2.

Akulenko, N. M., 2012. Haemopoietic system of anurans: the role of bone marrow and liver Vestnik zoologii. 46, e-28-e-35.

Andino, F. D. J., Chen, G., Li, Z., Grayfer, L., Robert, J., 2012. Susceptibility of Xenopus laevis tadpoles to infection by the ranavirus Frog-Virus 3 correlates with a reduced and delayed innate immune response in comparison with adult frogs. Virology. 432, 435-43.

Bartelmez, S. H., Bradley, T. R., Bertoncello, I., Mochizuki, D. Y., Tushinski, R. J., Stanley, E. R., Hapel, A. J., Young, I. G., Kriegler, A. B., Hodgson, G. S., 1989. Interleukin 1 plus interleukin 3 plus colony-stimulating factor 1 are essential for clonal proliferation of primitive myeloid bone marrow cells. Exp Hematol. 17, 240-5.

Baud'huin, M., Renault, R., Charrier, C., Riet, A., Moreau, A., Brion, R., Gouin, F., Duplomb, L., Heymann, D., 2010. Interleukin-34 is expressed by giant cell tumours of bone and plays a key role in RANKL-induced osteoclastogenesis. J Pathol. 221, 77-86.

Belosevic, M., Hanington, P. C., Barreda, D. R., 2006. Development of goldfish macrophages in vitro. Fish Shellfish Immunol. 20, 152-71.

Bober, L. A., Grace, M. J., Pugliese-Sivo, C., Rojas-Triana, A., Sullivan, L. M., Narula, S. K., 1995. The effects of colony stimulating factors on human monocyte cell function. Int $J$ Immunopharmacol. 17, 385-92.

Bonifer, C., Hume, D. A., 2008. The transcriptional regulation of the Colony-Stimulating Factor 1 Receptor (csf1r) gene during hematopoiesis. Front Biosci. 13, 549-60.

Brunst, V. V., 1958. The effect of total-body x-irradiation on the adult axolotl (Siredon mexicanum). Radiat Res. 8, 32-45.

Carpenter, K. L., Turpen, J. B., 1979. Experimental studies on hemopoiesis in the pronephros of Rana pipiens. Differentiation. 14, 167-74.

Carver, F. J., Meints, R. H., 1977. Studies of the development of frog hemopoietic tissue in vitro. I. Spleen culture assay of an erythropoietic factor in anemic frog blood. J Exp Zool. 201, 37-46.

Cecchini, M. G., Dominguez, M. G., Mocci, S., Wetterwald, A., Felix, R., Fleisch, H., Chisholm, O., Hofstetter, W., Pollard, J. W., Stanley, E. R., 1994. Role of colony stimulating factor1 in the establishment and regulation of tissue macrophages during postnatal development of the mouse. Development. 120, 1357-72.

Chemel, M., Le Goff, B., Brion, R., Cozic, C., Berreur, M., Amiaud, J., Bougras, G., Touchais, S., Blanchard, F., Heymann, M. F., Berthelot, J. M., Verrecchia, F., Heymann, D., 2012. Interleukin 34 expression is associated with synovitis severity in rheumatoid arthritis patients. Ann Rheum Dis. 71, 150-4.

Chen, X. D., Turpen, J. B., 1995. Intraembryonic origin of hepatic hematopoiesis in Xenopus laevis. J Immunol. 154, 2557-67.

Chen, Z., Buki, K., Vaaraniemi, J., Gu, G., Vaananen, H. K., 2011. The critical role of IL-34 in osteoclastogenesis. PLoS One. 6, e18689. 
Chihara, T., Suzu, S., Hassan, R., Chutiwitoonchai, N., Hiyoshi, M., Motoyoshi, K., Kimura, F., Okada, S., 2010. IL-34 and M-CSF share the receptor Fms but are not identical in biological activity and signal activation. Cell Death Differ. 17, 1917-27.

Chinchar, V. G., 2002. Ranaviruses (family Iridoviridae): emerging cold-blooded killers. Arch Virol. 147, 447-70.

Chinchar, V. G., Hyatt, A., Miyazaki, T., Williams, T., 2009. Family Iridoviridae: poor viral relations no longer. Curr Top Microbiol Immunol. 328, 123-70.

Ciau-Uitz, A., Monteiro, R., Kirmizitas, A., Patient, R., 2014. Developmental hematology: ontogeny, genetic programming and conservation. Exp Hematol. 42, 669-83.

Ciau-Uitz, A., Liu, F., Patient, R., 2010. Genetic control of hematopoietic development in Xenopus and zebrafish. Int J Dev Biol. 54, 1139-49.

Coiras, M., Lopez-Huertas, M. R., Perez-Olmeda, M., Alcami, J., 2009. Understanding HIV-1 latency provides clues for the eradication of long-term reservoirs. Nat Rev Microbiol. 7, 798-812.

Dai, X. M., Ryan, G. R., Hapel, A. J., Dominguez, M. G., Russell, R. G., Kapp, S., Sylvestre, V., Stanley, E. R., 2002. Targeted disruption of the mouse colony-stimulating factor 1 receptor gene results in osteopetrosis, mononuclear phagocyte deficiency, increased primitive progenitor cell frequencies, and reproductive defects. Blood. 99, 111-20.

de Abreu Manso, P. P., de Brito-Gitirana, L., Pelajo-Machado, M., 2009. Localization of hematopoietic cells in the bullfrog (Lithobates catesbeianus). Cell Tissue Res. 337, 30112.

Droin, N., Solary, E., 2010. Editorial: CSF1R, CSF-1, and IL-34, a "menage a trois" conserved across vertebrates. J Leukoc Biol. 87, 745-7.

Durand, C., Charlemagne, J., Fellah, J. S., 2000. RAG expression is restricted to the first year of life in the Mexican axolotl. Immunogenetics. 51, 681-7.

Eda, H., Shimada, H., Beidler, D. R., Monahan, J. B., 2011. Proinflammatory cytokines, IL1beta and TNF-alpha, induce expression of interleukin-34 mRNA via JNK- and p44/42 MAPK-NF-kappaB pathway but not p38 pathway in osteoblasts. Rheumatol Int. 31, 1525-30.

Flajnik, M.F., Hsu, E., Kaufman, J.F., Du Pasquier, L., 1987. Changes in the immune system during metamorphosis of Xenopus. Immunol Today 8, 58-64.

Frank, G., 1988. Granulopoiesis in tadpoles of Rana esculenta. Survey of the organs involved. $J$ Anat. 160, 59-66.

Frank, G., 1989. Granulopoiesis in tadpoles of Rana esculenta. Ultrastructural observations on the developing granulocytes and on the development of eosinophil granules. J Anat. 163, 97-105.

Garceau, V., Smith, J., Paton, I. R., Davey, M., Fares, M. A., Sester, D. P., Burt, D. W., Hume, D. A., 2010. Pivotal Advance: Avian colony-stimulating factor 1 (CSF-1), interleukin-34 (IL-34), and CSF-1 receptor genes and gene products. J Leukoc Biol. 87, 753-64.

Godwin, J.W., Rosenthal, N., 2014. Scar-free wound healing and regeneration in amphibians: immunological influences on regenerative success. Differentiation 87, 66-75.

Golub, R., Andre, S., Hassanin, A., Affaticati, P., Larijani, M., Fellah, J. S., 2004. Early expression of two TdT isoforms in the hematopoietic system of the Mexican axolotl. Implications for the evolutionary origin of the $\mathrm{N}$-nucleotide addition. Immunogenetics. 56, 204-13. 
Goodenow, M. M., Rose, S. L., Tuttle, D. L., Sleasman, J. W., 2003. HIV-1 fitness and macrophages. J Leukoc Biol. 74, 657-66.

Gordon, S., Martinez, F. O., 2010. Alternative activation of macrophages: mechanism and functions. Immunity. 32, 593-604.

Gordon, S., Taylor, P. R., 2005. Monocyte and macrophage heterogeneity. Nat Rev Immunol. 5, 953-64.

Gousset, K., Ablan, S. D., Coren, L. V., Ono, A., Soheilian, F., Nagashima, K., Ott, D. E., Freed, E. O., 2008. Real-time visualization of HIV-1 GAG trafficking in infected macrophages. PLoS Pathog. 4, e1000015.

Gow, D. J., Garceau, V., Kapetanovic, R., Sester, D. P., Fici, G. J., Shelly, J. A., Wilson, T. L., Hume, D. A., 2012. Cloning and expression of porcine Colony Stimulating Factor-1 (CSF-1) and Colony Stimulating Factor-1 Receptor (CSF-1R) and analysis of the species specificity of stimulation by CSF-1 and Interleukin 34. Cytokine. 60, 793-805.

Gow, D. J., Garceau, V., Pridans, C., Gow, A. G., Simpson, K. E., Gunn-Moore, D., Hume, D. A., 2013. Cloning and expression of feline colony stimulating factor receptor (CSF-1R) and analysis of the species specificity of stimulation by colony stimulating factor-1 (CSF1) and interleukin-34 (IL-34). Cytokine. 61, 630-8.

Grayfer, L., Andino, F. D. J., Chen, G., Chinchar, G. V., Robert, J., 2012. Immune evasion strategies of ranaviruses and innate immune responses to these emerging pathogens. Viruses. 4, 1075-92.

Grayfer, L., Belosevic, M., 2009. Molecular characterization of novel interferon gamma receptor 1 isoforms in zebrafish (Danio rerio) and goldfish (Carassius auratus L.). Mol Immunol. 46, 3050-9.

Grayfer, L., Andino, F. D. J., Robert, J., 2014. The amphibian (Xenopus laevis) type I interferon response to Frog Virus 3: new insight into ranavirus pathogenicity. $J$ Virol.

Grayfer, L., Edholm, E. E., Robert, J., 2015. Mechanisms of amphibian macrophage development: characterization of the Xenopus laevis colony-stimulating factor-1 receptor. Int J Dev Biol. (in press).

Grayfer, L., Garcia, E. G., Belosevic, M., 2010. Comparison of macrophage antimicrobial responses induced by type II interferons of the goldfish (Carassius auratus L.). J Biol Chem. 285, 23537-47.

Grayfer, L., Robert, J., 2013. Colony-stimulating factor-1-responsive macrophage precursors reside in the amphibian (Xenopus laevis) bone marrow rather than the hematopoietic subcapsular liver. J Innate Immun. 5, 531-42.

Grayfer, L., Robert, J., 2014. Divergent antiviral roles of amphibian (Xenopus laevis) macrophages elicited by colony-stimulating factor-1 and interleukin-34 J Leuk Biol. doi: jlb.4AB0315-117RR

Grayfer, L., Robert, J., 2015. Distinct functional roles of amphibian (Xenopus laevis) colonystimulating factor-1 and interleukin-34 macrophages J Leuk Biol. 96: 1143-1153

Grayfer, L., Edholm, E.S., Andino, F. D. J., Chinchar, V.G., Robert, J., 2015 Ranavirus host immunity and immune evasion. In: Ranaviruses: lethal pathogens of ectothermic vertebrates. Gray MJ (ed) Springer.

Greter, M., Lelios, I., Pelczar, P., Hoeffel, G., Price, J., Leboeuf, M., Kundig, T. M., Frei, K., Ginhoux, F., Merad, M., Becher, B., 2012. Stroma-derived interleukin-34 controls the development and maintenance of langerhans cells and the maintenance of microglia. Immunity. 37, 1050-60. 
Groot, F., Welsch, S., Sattentau, Q. J., 2008. Efficient HIV-1 transmission from macrophages to T cells across transient virological synapses. Blood. 111, 4660-3.

Guiducci, C., Vicari, A. P., Sangaletti, S., Trinchieri, G., Colombo, M. P., 2005. Redirecting in vivo elicited tumor infiltrating macrophages and dendritic cells towards tumor rejection. Cancer Res. 65, 3437-46.

Guilbert, L. J., Stanley, E. R., 1980. Specific interaction of murine colony-stimulating factor with mononuclear phagocytic cells. J Cell Biol. 85, 153-9.

Hadji-Azimi, I., Coosemans, V., Canicatti, C., 1987. Atlas of adult Xenopus laevis hematology. Dev Comp Immunol. 11, 807-74.

Hadji-Azimi, I., Coosemans, V., Canicatti, C., 1990. B-lymphocyte populations in Xenopus laevis. Dev Comp Immunol. 14, 69-84.

Haislip, N. A., Gray, M. J., Hoverman, J. T., Miller, D. L., 2011. Development and disease: how susceptibility to an emerging pathogen changes through anuran development. PLoS One. 6, e22307.

Hamilton, T. A., Zhao, C., Pavicic, P. G., Jr., Datta, S., 2014. Myeloid colony-stimulating factors as regulators of macrophage polarization. Front Immunol. 5, 554.

Hanington, P. C., Wang, T., Secombes, C. J., Belosevic, M., 2007. Growth factors of lower vertebrates: characterization of goldfish (Carassius auratus L.) macrophage colonystimulating factor-1. J Biol Chem. 282, 31865-72.

Hodgkinson, J. W., Grayfer, L., Belosevic, M., 2015. Biology of bony fish macrophages. Biology. 4, 881-906

Hwang, S. J., Choi, B., Kang, S. S., Chang, J. H., Kim, Y. G., Chung, Y. H., Sohn, D. H., So, M. W., Lee, C. K., Robinson, W. H., Chang, E. J., 2012. Interleukin-34 produced by human fibroblast-like synovial cells in rheumatoid arthritis supports osteoclastogenesis. Arthritis Res Ther. 14, R14.

Joerink, M., Ribeiro, C. M., Stet, R. J., Hermsen, T., Savelkoul, H. F., Wiegertjes, G. F., $2006 a$. Head kidney-derived macrophages of common carp (Cyprinus carpio L.) show plasticity and functional polarization upon differential stimulation. $J$ Immunol. 177, 61-9.

Joerink, M., Savelkoul, H. F., Wiegertjes, G. F., 2006b. Evolutionary conservation of alternative activation of macrophages: structural and functional characterization of arginase 1 and 2 in carp (Cyprinus carpio L.). Mol Immunol. 43, 1116-28.

Karbassi, A., Becker, J. M., Foster, J. S., Moore, R. N., 1987. Enhanced killing of Candida albicans by murine macrophages treated with macrophage colony-stimulating factor: evidence for augmented expression of mannose receptors. J Immunol. 139, 417-21.

Koths, K., 1997. Structure-function studies on human macrophage colony-stimulating factor (MCSF). Mol Reprod Dev. 46, 31-7; discussion 37-8.

Kriegler, A. B., Verschoor, S. M., Bernardo, D., Bertoncello, I., 1994. The relationship between different high proliferative potential colony-forming cells in mouse bone marrow. Exp Hematol. 22, 432-40.

Krysinska, H., Hoogenkamp, M., Ingram, R., Wilson, N., Tagoh, H., Laslo, P., Singh, H., Bonifer, C., 2007. A two-step, PU.1-dependent mechanism for developmentally regulated chromatin remodeling and transcription of the c-fms gene. Mol Cell Biol. 27, 878-87.

Lane, M. C., Sheets, M. D., 2002. Primitive and definitive blood share a common origin in Xenopus: a comparison of lineage techniques used to construct fate maps. Dev Biol. 248, $52-67$. 
Lichanska, A. M., Browne, C. M., Henkel, G. W., Murphy, K. M., Ostrowski, M. C., McKercher, S. R., Maki, R. A., Hume, D. A., 1999. Differentiation of the mononuclear phagocyte system during mouse embryogenesis: the role of transcription factor PU.1. Blood. 94, 127-38.

Lin, H., Lee, E., Hestir, K., Leo, C., Huang, M., Bosch, E., Halenbeck, R., Wu, G., Zhou, A., Behrens, D., Hollenbaugh, D., Linnemann, T., Qin, M., Wong, J., Chu, K., Doberstein, S. K., Williams, L. T., 2008. Discovery of a cytokine and its receptor by functional screening of the extracellular proteome. Science. 320, 807-11.

Liu, H., Leo, C., Chen, X., Wong, B. R., Williams, L. T., Lin, H., He, X., 2014. The mechanism of shared but distinct CSF-1R signaling by the non-homologous cytokines IL-34 and CSF-1. Biochim Biophys Acta. 1824, 938-45.

Lopez, D., Lin, L., Monaghan, J. R., Cogle, C. R., Bova, F. J., Maden, M., Scott, E. W., 2014. Mapping hematopoiesis in a fully regenerative vertebrate: the axolotl. Blood. 124, 123241.

Ma, X., Lin, W. Y., Chen, Y., Stawicki, S., Mukhyala, K., Wu, Y., Martin, F., Bazan, J. F., Starovasnik, M. A., 2012. Structural basis for the dual recognition of helical cytokines IL-34 and CSF-1 by CSF-1R. Structure. 20, 676-87.

Manos, M. M., 1988. Expression and processing of a recombinant human macrophage colonystimulating factor in mouse cells. Mol Cell Biol. 8, 5035-9.

Mantovani, A., Sozzani, S., Locati, M., Allavena, P., Sica, A., 2002. Macrophage polarization: tumor-associated macrophages as a paradigm for polarized M2 mononuclear phagocytes. Trends Immunol. 23, 549-55.

Meseguer, J., Lozano, M. T., Agulleiro, B., 1985. Ultrastructure of the renal granulopoietic tissue of the Rana ridibunda tadpole. J Submicrosc Cytol. 17, 391-401.

Marr, S., Morales, H., Bottaro, A., Cooper, M., Flajnik, M., Robert, J., 2007. Localization and differential expression of activation-induced cytidine deaminase in the amphibian Xenopus upon antigen stimulation and during early development. J Immunol. 179, 678389.

Mills, C. D., 2012. M1 and M2 Macrophages: Oracles of Health and Disease. Crit Rev Immunol. 32, 463-88.

Morales, H. D., Abramowitz, L., Gertz, J., Sowa, J., Vogel, A., Robert, J., 2010. Innate immune responses and permissiveness to ranavirus infection of peritoneal leukocytes in the frog Xenopus laevis. J Virol. 84, 4912-22.

Munn, D. H., Cheung, N. K., 1995. Antibody-independent phagocytosis of tumor cells by human monocyte-derived macrophages cultured in recombinant macrophage colony-stimulating factor. Cancer Immunol Immunother. 41, 46-52.

Neumann, N. F., Barreda, D. R., Belosevic, M., 2000. Generation and functional analysis of distinct macrophage sub-populations from goldfish (Carassius auratus L.) kidney leukocyte cultures. Fish Shellfish Immunol. 10, 1-20.

Nishikawa, A., Murata, E., Akita, M., Kaneko, K., Moriya, O., Tomita, M., Hayashi, H., 1998. Roles of macrophages in programmed cell death and remodeling of tail and body muscle of Xenopus laevis during metamorphosis. Histochem Cell Biol. 109, 11-7.

Nogawa-Kosaka, N., Sugai, T., Nagasawa, K., Tanizaki, Y., Meguro, M., Aizawa, Y., Maekawa, S., Adachi, M., Kuroki, R., Kato, T., 2011. Identification of erythroid progenitors induced by erythropoietic activity in Xenopus laevis. J Exp Biol. 214, 921-7. 
Paredes, R., Ishibashi, S., Borrill, R., Robert J., Amaya, E., 2015. Xenopus larvae: an easily tractable model for live investigation of the inflammatory response following injury or bacterial infection. Dev. Biol., Special issue on Xenopus. pii: S0012-1606(15)00121-9

Pixley, F. J., Stanley, E. R., 2004. CSF-1 regulation of the wandering macrophage: complexity in action. Trends Cell Biol. 14, 628-38.

Rettenmier, C. W., Roussel, M. F., 1988. Differential processing of colony-stimulating factor 1 precursors encoded by two human cDNAs. Mol Cell Biol. 8, 5026-34.

Robert, J., Abramowitz, L., Gantress, J., Morales, H. D., 2007. Xenopus laevis: a possible vector of Ranavirus infection? J Wildl Dis. 43, 645-52.

Robert, J., Grayfer, L., Edholm, E. S., Ward, B., Andino, F. D. J. 2014. Inflammation-induced reactivation of the ranavirus Frog Virus 3 in asymptomatic Xenopus laevis. PLoS One. 9, e112904.

Robert, J., Ohta, Y. 2009. Comparative and developmental study of the immune system in Xenopus. Dev. Dyn. 238, 1249-70.

Rosa, A., Ballarino, M., Sorrentino, A., Sthandier, O., De Angelis, F. G., Marchioni, M., Masella, B., Guarini, A., Fatica, A., Peschle, C., Bozzoni, I., 2007. The interplay between the master transcription factor PU.1 and miR-424 regulates human monocyte/macrophage differentiation. Proc Natl Acad Sci U S A. 104, 19849-54.

Saccani, A., Schioppa, T., Porta, C., Biswas, S. K., Nebuloni, M., Vago, L., Bottazzi, B., Colombo, M. P., Mantovani, A., Sica, A., 2006. p50 nuclear factor-kappaB overexpression in tumor-associated macrophages inhibits M1 inflammatory responses and antitumor resistance. Cancer Res. 66, 11432-40.

Shibasaki, Y., Yabu, T., Araki, K., Mano, N., Shiba, H., Moritomo, T., Nakanishi, T., 2013. Peculiar monomeric interferon gammas, IFNgammarel 1 and IFNgammarel 2, in ginbuna crucian carp. Febs $J$.

Stanley, E. R., Berg, K. L., Einstein, D. B., Lee, P. S., Pixley, F. J., Wang, Y., Yeung, Y. G., 1997. Biology and action of colony--stimulating factor-1. Mol Reprod Dev. 46, 4-10.

Stanley, E. R., Chen, D. M., Lin, H. S., 1978. Induction of macrophage production and proliferation by a purified colony stimulating factor. Nature. 274, 168-70.

Sweet, M. J., Hume, D. A., 2003. CSF-1 as a regulator of macrophage activation and immune responses. Arch Immunol Ther Exp (Warsz). 51, 169-77.

Tagoh, H., Himes, R., Clarke, D., Leenen, P. J., Riggs, A. D., Hume, D., Bonifer, C., 2002. Transcription factor complex formation and chromatin fine structure alterations at the murine c-fms (CSF-1 receptor) locus during maturation of myeloid precursor cells. Genes Dev. 16, 1721-37.

Tanaka, Y., 1976. Architecture of the marrow vasculature in three amphibian species and its significance in hematopoietic development. Am J Anat. 145, 485-97.

Taylor, E. W., Fear, A. L., Bohm, A., Kim, S. H., Koths, K., 1994. Structure-function studies on recombinant human macrophage colony-stimulating factor (M-CSF). J Biol Chem. 269, 31171-7.

Turpen, J. B., Turpen, C. J., Flajnik, M., 1979. Experimental analysis of hematopoietic cell development in the liver of larval Rana pipiens. Dev Biol. 69, 466-79.

Tushinski, R. J., Oliver, I. T., Guilbert, L. J., Tynan, P. W., Warner, J. R., Stanley, E. R., 1982. Survival of mononuclear phagocytes depends on a lineage-specific growth factor that the differentiated cells selectively destroy. Cell. 28, 71-81. 
Venkatesh, B., Lee, A. P., Ravi, V., Maurya, A. K., Lian, M. M., Swann, J. B., Ohta, Y., Flajnik, M. F., Sutoh, Y., Kasahara, M., Hoon, S., Gangu, V., Roy, S. W., Irimia, M., Korzh, V., Kondrychyn, I., Lim, Z. W., Tay, B. H., Tohari, S., Kong, K. W., Ho, S., Lorente-Galdos, B., Quilez, J., Marques-Bonet, T., Raney, B. J., Ingham, P. W., Tay, A., Hillier, L. W., Minx, P., Boehm, T., Wilson, R. K., Brenner, S., Warren, W. C., 2014. Elephant shark genome provides unique insights into gnathostome evolution. Nature. 505, 174-9.

Wang, N., Liang, H., Zen, K., 2014. Molecular mechanisms that influence the macrophage m1$\mathrm{m} 2$ polarization balance. Front Immunol. 5, 614.

Wang, T., Hanington, P. C., Belosevic, M., Secombes, C. J., 2008. Two macrophage colonystimulating factor genes exist in fish that differ in gene organization and are differentially expressed. J Immunol. 181, 3310-22.

Wang, T., Kono, T., Monte, M. M., Kuse, H., Costa, M. M., Korenaga, H., Maehr, T., Husain, M., Sakai, M., Secombes, C. J., 2013. Identification of IL-34 in teleost fish: differential expression of rainbow trout IL-34, MCSF1 and MCSF2, ligands of the MCSF receptor. Mol Immunol. 53, 398-409.

Wang, Y., Colonna, M., 2014. Interkeukin-34, a cytokine crucial for the differentiation and maintenance of tissue resident macrophages and Langerhans cells. Eur J Immunol.

Wei, S., Nandi, S., Chitu, V., Yeung, Y. G., Yu, W., Huang, M., Williams, L. T., Lin, H., Stanley, E. R., 2010. Functional overlap but differential expression of CSF-1 and IL-34 in their CSF-1 receptor-mediated regulation of myeloid cells. J Leukoc Biol. 88, 495-505.

Williams, H., Brenner, S., Venkatesh, B., 2002. Identification and analysis of additional copies of the platelet-derived growth factor receptor and colony stimulating factor 1 receptor genes in fugu. Gene. 295, 255-64.

Williams, T., Barbosa-Solomieu, V., Chinchar, V. G., 2005. A decade of advances in iridovirus research. Adv Virus Res. 65, 173-248.

Yabu, T., Toda, H., Shibasaki, Y., Araki, K., Yamashita, M., Anzai, H., Mano, N., Masuhiro, Y., Hanazawa, S., Shiba, H., Moritomo, T., Nakanishi, T., 2011. Antiviral protection mechanisms mediated by ginbuna crucian carp interferon gamma isoforms 1 and 2 through two distinct interferon gamma-receptors. J Biochem. 150, 635-48.

Yamane, F., Nishikawa, Y., Matsui, K., Asakura, M., Iwasaki, E., Watanabe, K., Tanimoto, H., Sano, H., Fujiwara, Y., Stanley, E. R., Kanayama, N., Mabbott, N. A., Magari, M., Ohmori, H., 2014. CSF-1 receptor-mediated differentiation of a new type of monocytic cell with B cell-stimulating activity: its selective dependence on IL-34. J Leukoc Biol. 95, 19-31.

Zhou, D., Huang, C., Lin, Z., Zhan, S., Kong, L., Fang, C., Li, J., 2013. Macrophage polarization and function with emphasis on the evolving roles of coordinated regulation of cellular signaling pathways. Cell Signal. 26, 192-7. 